\title{
Predicting the ideal Valve Size during Aortic Valve Replacement with Rapid Deployment Bioprosthetic Valves. Is Intraoperative Transesophageal Echocardiography reliable?
}

\author{
Ali Al-Alameri ${ }^{1}$, Alejandro Macias ${ }^{2}$, Evan Markell ${ }^{3}$, Julio Benitez ${ }^{3}$, Alvaro Montoya ${ }^{3}$, and \\ Michael Magarakis ${ }^{3}$ \\ ${ }^{1}$ Jackson Memorial Hospital \\ ${ }^{2}$ University of Miami Health System \\ ${ }^{3}$ Miami VA Medical Center - University of Miami Miller School of Medicine
}

May 15, 2020

\begin{abstract}
Sutureless/rapid deployment valves provide surgeons with a new tool for aortic valve replacement (AVR) therapy, which can simplify the procedure. A main concern being the development of a paravalvular leak. We retrospectively reviewed our Aortic Valve Replacement database, were able to identify 25 rapid deployment aortic valves (INTUITY Valve System, Edwards Life Sciences LLC, Irvine, Calif) that had documented intraoperative Transesophageal Echocardiographic Annular measurements. All valves were implanted in the Aortic position. In this cohort, only patients that had their native aortic valve replaced either isolated or as part of any additional procedure were included. Patients that underwent Intuity valve implantation for Redo Aortic valve replacement were excluded from this study. After review of 25 patients who underwent rapid deployment valve implantation in the aortic position, $36 \%$ of patients had the same size valve as the measured aortic annulus, $48 \%$ of patients had a valve implanted that was $1 \mathrm{~mm}$ different, and $16 \%$ of patients had $2 \mathrm{~mm}$ difference. The mean annular size based on intraoperative TEE was $22.4 \mathrm{~mm}$ (range: $21-28 \mathrm{~mm}$ ). The mean valve size that we implanted was $23.3 \mathrm{~mm}$ (range: $21-27 \mathrm{~mm}$ ). No statistically significant difference between the mean annular measurement and the valve size selected $(0.9 \mathrm{~mm}, \mathrm{p}=0.8)$ were found. TEE is perhaps remains as one of our most important tool to further enhance valve sizing and appropriately guide one through a proper and safe deployment.
\end{abstract}

\section{Hosted file}

Intuity Valve Sizing TEE.docx available at https://authorea.com/users/322282/articles/451414predicting-the-ideal-valve-size-during-aortic-valve-replacement-with-rapid-deploymentbioprosthetic-valves-is-intraoperative-transesophageal-echocardiography-reliable 


\begin{tabular}{|c|c|}
\hline \multicolumn{2}{|l|}{ Age } \\
\hline \multicolumn{2}{|c|}{$>55-75(68.3)$} \\
\hline \multicolumn{2}{|c|}{ Diagnosis } \\
\hline AS & $11 / 25$ \\
\hline$A S+C A D$ & $9 / 25$ \\
\hline Al+ATAA & 2/25 \\
\hline$A S+M R$ & $1 / 25$ \\
\hline$A l+C A D$ & $1 / 25$ \\
\hline \multicolumn{2}{|c|}{ Approach } \\
\hline Sternotomy & $21 / 25$ \\
\hline Upper sternotomy & 2/25 \\
\hline Rt thoracotomy & $2 / 25$ \\
\hline \multicolumn{2}{|c|}{ Procedure } \\
\hline \multicolumn{2}{|c|}{ Isolated AVR 11/25 } \\
\hline \multicolumn{2}{|c|}{ AVR+CABG $11 / 25$} \\
\hline \multicolumn{2}{|c|}{ AVR+MVR $1 / 25$} \\
\hline \multicolumn{2}{|c|}{ AVR+Ascending root replacement $2 / 25$} \\
\hline \multicolumn{2}{|c|}{ Outcomes } \\
\hline \multicolumn{2}{|c|}{30 days Mortality $0 / 25$} \\
\hline \multicolumn{2}{|c|}{30 days stroke $0 / 25$} \\
\hline \multicolumn{2}{|c|}{ Major bleeding 0/25 } \\
\hline \multicolumn{2}{|c|}{ Valve thrombosis $0 / 25$} \\
\hline \multicolumn{2}{|c|}{ Valve explant $0 / 25$} \\
\hline \multicolumn{2}{|c|}{ Major post op PVL $0 / 25$} \\
\hline \multicolumn{2}{|c|}{ Trace post op PVL 5} \\
\hline
\end{tabular}




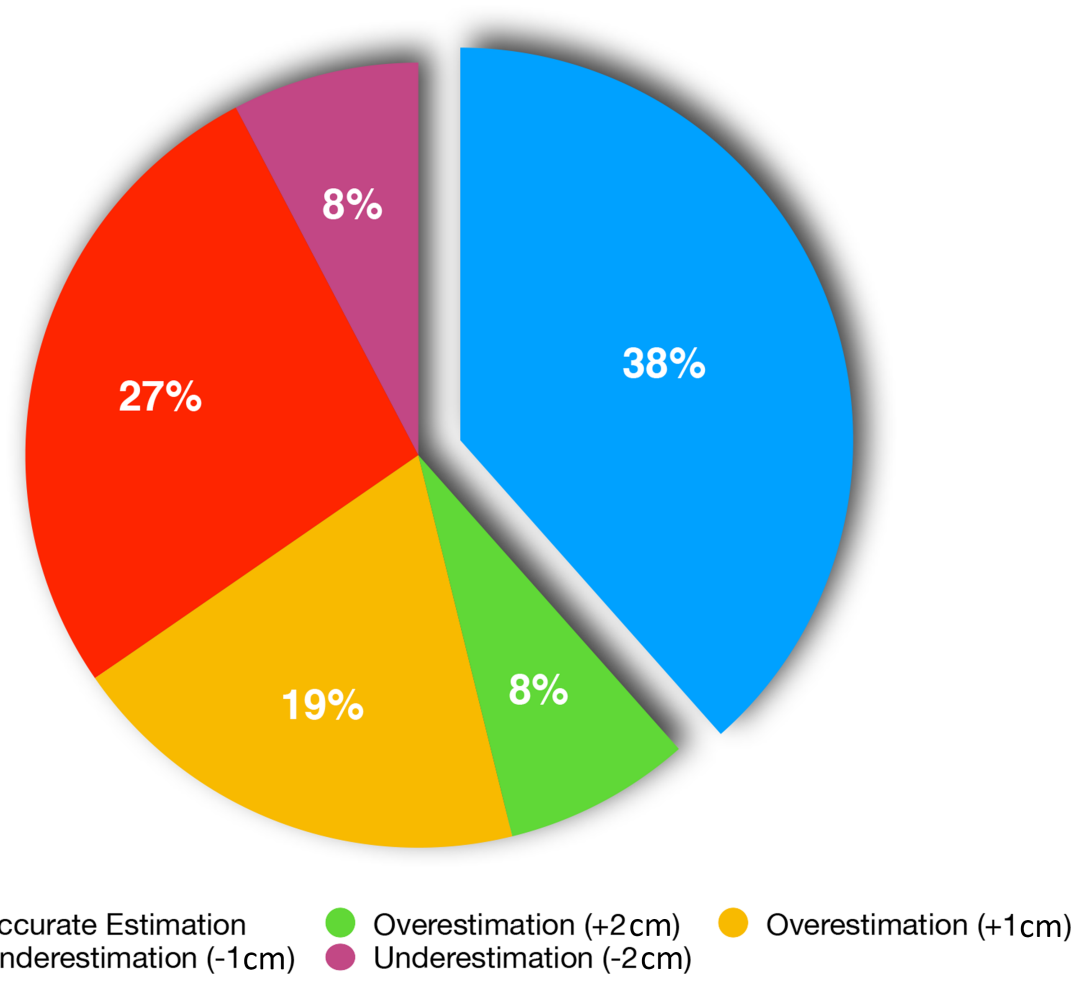

Accurate Estimation

Underestimation $(-2 \mathrm{~cm})$ 